\title{
FINANCIAMENTO PÚBLICO À INOVAÇÃO NO SETOR FARMACÊUTICO BRASILEIRO E A INCORPORAÇÃO DE TECNOLOGIAS NO SUS
}

\author{
Luiza Pinheiro Alves da Silva (Doutoranda em Inovação Tecnológica e Biofarmacêutica- \\ UFMG) \\ Márcia Siqueira Rapini (CEDEPLAR- UFMG)
}

Área ABEIN 6: 6.2 - Políticas de Ciência, Tecnologia e Inovação

\section{RESUMO}

O desenvolvimento de novas tecnologias em saúde tem potencial para contribuir para a melhoria das condições de saúde de uma população, para ampliar o conhecimento científico e tecnológico, e para promover o crescimento econômico e a competitividade do país no cenário internacional. O objetivo deste artigo foi analisar o alinhamento entre ações voltadas para o desenvolvimento de novas tecnologias no setor farmacêutico e a incorporação destas tecnologias no sistema público de saúde brasileiro. Foram comparados os processos e critérios para a seleção de projetos no programa de Subvenção Econômica da FINEP e os critérios para incorporação no SUS adotados pela CONITEC. Observou-se pontos de convergência, no entanto, é recomendável um alinhamento mais estreito entre as agências de fomento e o SUS, de modo a favorecer um maior desenvolvimento tecnológico, econômico e social.

PALAVRAS- CHAVE: Políticas de Ciência, Tecnologia e Inovação; financiamento público; Sistema Único de Saúde.

\begin{abstract}
The development of new technologies in health has the potential to contribute to the improvement of the health conditions of a population and to the expansion of scientific and technological knowledge, as well as to promote economic growth and the competitiveness of a country in the international scenario. The objective of this article was to analyze the alignment between actions aimed at the development of new technologies in the pharmaceutical sector, and actions to incorporate these technologies in the Brazilian public health system. The criteria for the selection of projects in FINEP's Subvenção Econômica program were compared with the criteria for incorporation adopted by CONITEC. Points of convergence were observed, however, a closer alignment between development agencies and SUS is recommended, in order to guarantee the achievement of greater technological, economic and social development.
\end{abstract}

KEY WORDS: Science, Technology and Innovation policies; public funding; Unified Health System.

\section{JEL Code: 038}




\section{INTRODUÇÃO}

O desenvolvimento de novas tecnologias em saúde tem potencial não apenas para contribuir para a melhoria das condições de saúde de uma população e para ampliar o conhecimento científico e tecnológico, mas também para promover o crescimento econômico e a competitividade do país no cenário internacional (GUIMARÃES, 2006; NOVAES; ELIAS, 2013). A necessidade da articulação entre produção-incorporaçãoutilização de tecnologias é importante para o fortalecimento da capacitação tecnológica de um país, e é ainda mais necessária nos países em desenvolvimento. Além dos fatores de preocupação comuns aos observados nos países desenvolvidos, como o fato das novas tecnologias em saúde terem um impacto nos orçamentos de saúde cada vez maior e seu uso ser cumulativo e da mudança de perfil epidemiológico com o envelhecimento da população, os países em desenvolvimento sofrem com o aumento contínuo do déficit da balança comercial do setor saúde. Além disto, nestes países o sistema produtivo local não atende as demandas tecnológicas nacionais nesta área , seja por não ser capaz de produzir grande parte dos medicamentos já utilizados no sistema de saúde, seja porque não é capaz de desenvolver medicamentos que atendam às lacunas deixadas pela indústria multinacional. Em ambos os casos, a falta de capacitação tecnológica da indústria local é um elemento-chave para explicar a incapacidade de criação de melhorias ou adaptações de tecnologias já existentes e para o desenvolvimento de tecnologias disruptivas mais adequadas às necessidades locais (KLEIN; HASENCLEVER; MACHADO, 2011)

$\mathrm{O}$ estímulo às inovações neste setor é ligado a ações de bem-estar social e de desenvolvimento econômico, sendo alvo tanto de políticas de saúde pública quanto de políticas industriais e de inovação, mesmo que estas não sejam construídas de forma coordenada entre si. Além disto, a exploração de tecnologias em saúde se caracteriza por gerar grandes assimetrias, em muitos casos promovendo a apropriação privada de recursos coletivos como o conhecimento e o progresso técnico, e a exclusão de indivíduos, regiões e países.(GADELHA, 2006) Assim, cabe ao Estado não só fomentar o desenvolvimento de inovações, mas promover o acesso igualitário e racional a elas, principalmente em sistemas universais de saúde, nos quais parte crítica do orçamento é dedicada à aquisição e ao uso de novas tecnologias. Refletindo a complexidade deste cenário, o Sistema de Inovação em Saúde envolve atores de naturezas diversas.

Neste contexto, o presente trabalho buscou identificar e comparar os processos de avaliação e os critérios adotados por uma agência federal de fomento à inovação, a Empresa Brasileira de Inovação e Pesquisa - FINEP ${ }^{1}$, para a seleção de projetos de desenvolvimento de novas tecnologias a serem financiados na área de saúde com recursos de subvenção econômica, e os critérios utilizados pela Comissão Nacional de Incorporação de Tecnologias no SUS - CONITEC para incorporação de tecnologias no sistema público de saúde brasileiro.

A FINEP foi escolhida por ser uma das principais agências de fomento à inovação no país, aportando recursos tanto em instituições científicas e tecnológicas quanto em empresas. O setor farmacêutico vem sendo considerado prioritário nas três últimas Políticas Industrias Brasileira, tendo recebido consideráveis aportes de recursos para a inovação da Finep e do BNDES (Teixeira, et. al, 2016). Dentre os diversos instrumentos

\footnotetext{
1 A FINEP era conhecida como Financiadora de Estudos e Projetos, depois passou a ser denominada Inovação e Pesquisa e em 2018 passou a ser Empresa Brasileira de Inovação e Pesquisa.
} 
de financiamento da FINEP, os editais Subvenção Econômica foram escolhidos para análise por serem um dos principais instrumentos de fomento à inovação do governo federal, pois disponibilizam recursos não reembolsáveis, sendo assim um dos mais visados pelas empresas, independente do seu porte. Além disto, este tipo de recurso é orientado para projetos com alto grau de incerteza, uma característica inerente ao setor farmacêutico já que seus projetos de inovação apresentam alto risco de desenvolvimento devido a diversos fatores tais como longo tempo de desenvolvimento, necessidade de grande volume de recursos e alta barreira regulatória.

Além desta breve introdução, o artigo possui mais 4 seções. A seção 2 discorre sobre a importância da articulação entre a Politica Industrial e a Política da Saúde e apresenta, brevemente, o Sistema Brasileiro Setorial de Inovação em Saúde com foco na atuação das duas instituições analisadas no trabalho: a FINEP e a CONITEC. A seção 3 descreve a metodologia utilizada para o desenvolvimento do artigo, e os resultados e discussão e a conclusão estão apresentados na seção 4 . A seção 5 conclui o trabalho.

\section{ARTICULAÇÃO ENTRE POLÍTICA INDUSTRIAL E DA POLÍTICA DA SAÚDE: UMA CONVERSA NECESSÁRIA.}

Um Sistema Nacional de Inovação é uma construção institucional, produto de uma ação planejada e consciente ou de um somatório de decisões não planejadas e desarticuladas, que impulsiona o progresso tecnológico em economias capitalistas complexas (ALBUQUERQUE, 1998). Devido a especificidades dos diferentes setores, tais como as bases de conhecimento, os atores envolvidos no processo de inovação, os elos e as relações entre estes atores e as instituições mais relevantes, é possível delinear sistemas setoriais de inovação (MALERBA, 2006). O Sistema Setorial de Inovação em Saúde Brasileiro ainda pode ser considerado como imaturo, necessitando desenvolver instituições e relações eficientes entre os agentes. (ALBUQUERQUE, 1998; GUIMARÃES, 2006)

Uma atividade determinante do sistema de inovação é o financiamento das atividades inovativas. $\mathrm{O}$ investimento em inovação possui peculiaridades que o difere de outros tipos de investimento, inclusive daqueles de longo prazo. Esses investimentos apresentam um elevado nível de incerteza associada ao processo inovativo. É altamente incerto prever o sucesso de um projeto de desenvolvimento de um novo produto ou processo, pois mesmo que se conheça os padrões que levaram a uma inovação prévia bem-sucedida, é muito difícil prever a realidade do mercado, as condições epidemiológicas e o comportamento dos concorrentes no momento de sua introdução no mercado, tampouco é possível se preparar antecipadamente. Esta barreira dificulta o financiamento à inovação, em especial em momentos de crise econômica. Além disto, outras barreiras do investimento a inovação são bem conhecidas: o conhecimento gerado é tácito, o retorno dos investimentos é demorado e incerto e existe uma grande assimetria de informações entre o inovador, disposto ao risco, e o investidor, avesso ao risco, bem como, no caso da saúde, entre o consumidor ou usuário, neste caso os pacientes, e o comprador da tecnologia, o sistema público de saúde ou as seguradoras de saúde privada. (LEONEL et al., 2013) Estas dificuldades se agravam no caso do investimento em tecnologias de saúde, em especial farmacêuticas, devido ao alto volume de recursos necessários, que envolvem 
mão de obra altamente qualificada, materiais e equipamentos de alto custo, tempo de desenvolvimento ainda mais longo que para outras tecnologias e exigências regulatórias rígidas desde a condução ética dos ensaios clínicos até o registro para a comercialização.

Um dos principais atores de qualquer sistema de inovação é o Estado, sendo um agente muito relevante no Sistema Setorial de Inovação em Saúde. Neste, o Estado tem papel fundamental, pois deve assegurar o direito da garantia à saúde da população e a obrigação de promover o desenvolvimento econômico, social e tecnológico. Suas atividades incluem não somente o fomento e financiamento as atividades inovativas, mas também a regulação sanitária e econômica, além do consumo, já que o Estado se apresenta como principal comprador de novas tecnologias em saúde. No caso brasileiro, o Estado possui a obrigação de garantir a eficácia e a segurança das tecnologias para a saúde utilizadas pela sociedade e também de garantir o acesso a essas tecnologias por meio do SUS, considerando um orçamento limitado no qual uma parcela significativa é dedicada à aquisição, distribuição e uso da tecnologia (VIANA; ELIAS, 2007).

Essa diversidade de papéis a serem empenhados se traduz na presença de diversos atores estatais atuando no Sistema de Inovação em Saúde, sendo alguns exemplos a Agência Nacional de Vigilância Sanitária (ANVISA), Agência Nacional de Saúde (ANS), no âmbito da saúde e a e o Banco Nacional de Desenvolvimento Econômico e Social (BNDES), no âmbito da política industrial e de inovação, sendo estas instituições capitaneadas pelo Ministério da Saúde (MS) e pelo Ministério da Ciência, Tecnologia , Inovações e Comunicação (MCTIC) ou Ministério do Desenvolvimento, Indústria e Comércio exterior (MDIC), além da CONITEC e da FINEP. Ademais, o desenvolvimento de novas tecnologias em saúde é objeto tanto de Políticas de Saúde pública quanto de Políticas Industriais e de Inovação e, apesar delas compartilharem os princípios de promoção da saúde para a população, bem como o desenvolvimento econômico e científico, muitas vezes a ação dos atores que executa estas políticas não é alinhada ou sinérgica.

Uma melhor articulação entre os agentes, fator importante para o amadurecimento do Sistema de Inovação, é citada por alguns autores como um dos pontos chaves para a melhoria do Sistema Setorial de Inovação em Saúde. Guimarães (2006) cita que o principal desafio para o parque de $\mathrm{P} \& \mathrm{D}$ em saúde no Brasil está associado ao amadurecimento do sistema setorial de inovação em saúde. Entre outros aspectos, isso significa o reforço às atividades de pesquisa em saúde nas universidades e institutos de pesquisa, ajustando-as a prioridades estabelecidas pelo sistema de saúde, em particular por seu componente público, além da construção de relações mais sólidas e permanentes entre estas instituições, as empresas e o sistema de saúde. (GUIMARÃES, 2006). Gadelha (2006) destaca entre temas importantes de análise o estudo da dinâmica industrial e de inovação nas indústrias da saúde e sua articulação com o sistema de atenção à saúde, bem como o esforço para introduzir mudanças institucionais no setor produtivo e nas instituições de suporte, sobretudo no âmbito financeiro e das organizações de pesquisa e de tecnologia, envolvendo uma forte transformação do próprio Estado. Gadelha (2006) defende ainda que é necessário acoplar uma nova agenda voltada para a concepção de políticas de desenvolvimento das atividades produtivas à agenda usual da pesquisa e da política de saúde. Ainda, Novaes e Elias (2013) afirmam que há também um reconhecimento crescente que para que os impactos da pesquisa em saúde se realizem são necessárias ações que promovam a articulação entre a produção científica e os diferentes setores sociais estratégicos. Capanema e Palmeira Filho, (2004) colocam que a política 
para estimular a produção de farmoquímicos e medicamentos deve ser estreitamente coordenada com a política de saúde e com as políticas de regulação, sob pena de serem invalidados os esforços para o desenvolvimento da indústria farmacêutica doméstica.

Para entender sua posição no Sistema Brasileiro de Inovação em Saúde e compreender sua articulação com outras agências estatais, é importante descrever o histórico das duas instituições que serão analisadas neste estudo, a FINEP e a CONITEC.

\subsection{FINEP}

A FINEP é um dos principais financiadores do Sistema de Brasileiro de Inovação. Foi criada em 1967 com a finalidade de financiar estudos e programas necessários à definição dos projetos de modernização e industrialização correntes na época. Em 1969 foi criado o Fundo Nacional de Desenvolvimento Científico e Tecnológico (FNDCT) com a finalidade de dar apoio financeiro aos programas e projetos prioritários de desenvolvimento científico e tecnológico. A FINEP atua como secretária executiva do FNDCT. (BRASIL, 2016a) Até hoje o FNDCT por meio da FINEP continua sendo um dos principais instrumentos que o Estado brasileiro usa para investimento em ciência, tecnologia e inovação.

Em 2004 foi aprovado o marco legal da inovação no Brasil, a Lei 10.973/04, chamada Lei da Inovação. Dentre outras medidas importantes de fomento à inovação, a Lei de Inovação instituiu a subvenção econômica, que são recursos dedicados exclusivamente ao financiamento de atividades de pesquisa, desenvolvimento tecnológico e inovação em empresas. Esta modalidade de financiamento é considerada importante para o estímulo da inovação em empresas por ser uma forma de compartilhamento dos custos e riscos inerentes a tais atividades e tem como objetivo promover um significativo aumento das atividades de inovação e o incremento da competitividade das empresas e da economia do País (BRASIL, 2016b). O primeiro edital de Subvenção Econômica foi lançado em 2006 pela FINEP, sendo lançados editais anualmente até 2010. Os editais seguintes foram lançados em 2013 e foram temáticos, havendo editais separados para os diferentes temas (BRASIL, 2016c)

De modo geral, os editais contêm as áreas priorizadas, os requisitos dos participantes, informações sobre os recursos disponíveis, as características da proposta e os critérios para sua seleção e um cronograma da chamada, além de disposições gerais. O processo de avaliação e os critérios de seleção variam de acordo com cada edital. De modo geral, primeiramente se faz uma análise documental, para checar se todas as exigências formais foram cumpridas, e em seguida a proposta é analisada por comitês de especialistas, tanto da FINEP quanto consultores externos. Em relação à avaliação da proposta em si, geralmente os requisitos abrangem critérios pertinentes ao mérito da inovação, aos seus aspectos mercadológicos e à capacidade de execução e os aportes da empresa.

\subsection{CONITEC}

Já a CONITEC é um dos agentes reguladores do Sistema Brasileiro de Inovação em saúde, já que decide sobre as tecnologias a serem incorporadas, e logo disponibilizadas pelo SUS. A avaliação de tecnologias para a incorporação destas no sistema público de saúde surgiu da necessidade de avaliar a eficácia das novas opções terapêuticas disponibilizadas juntamente com a necessidade de se otimizar a alocação dos recursos limitados para saúde, visto a amplitude de necessidades e os custos crescentes nesta área.(NOVAES; ELIAS, 2013) 
O assunto ganhou força no Ministério da Saúde a partir de 2003 com a criação de um grupo de trabalho no âmbito do Conselho de Ciência, Tecnologia e Inovação do Ministério da Saúde. Em 2006 o fluxo para incorporação de tecnologias no SUS é normatizado pela primeira vez, e foi criada a Comissão de Incorporação de Tecnologias (CITEC) inicialmente ligada a Secretaria de Atenção a Saúde (SAS) mas em 2008 é transferida para a Secretaria de Ciência, Tecnologia e Insumos Estratégicos (SCTIE). Em 2011 a CITEC é substituída pela Comissão Nacional de Incorporação de Tecnologias no SUS (CONITEC), e as mudanças ocorridas então incluem uma nova composição do plenário da Comissão, ampliando a participação da sociedade e do próprio Ministério da Saúde, a definição de prazos para o processo de análise e para a disponibilização pelo SUS da nova tecnologia. As competências da CONITEC são análogas às da CITEC e incluem assessorar o MS na incorporação, exclusão ou alteração pelo SUS de novas tecnologias em saúde, como medicamentos, produtos e procedimentos, tais como vacinas, produtos para diagnóstico de uso "in vitro", equipamentos, procedimentos técnicos, sistemas organizacionais, informacionais, educacionais e de suporte, programas e protocolos assistenciais, por meio dos quais a atenção e os cuidados com a saúde são prestados à população, constituir e alterar protocolos clínicos e diretrizes terapêuticas, podendo requisitar a sua elaboração ou revisão, conforme o interesse para o SUS, sendo que os protocolos clínicos e as diretriz terapêutica estabelecem critérios para o diagnóstico da doença ou do agravo à saúde; o tratamento preconizado, os medicamentos e demais produtos apropriados, quando couber; as posologias recomendadas; os mecanismos de controle clínico; e o acompanhamento e a verificação dos resultados terapêuticos, a serem seguidos pelos gestores do SUS. (BRASIL, 2016d)

A CONITEC é composta por dois fóruns: a Secretaria-Executiva e o Plenário. A Secretaria-Executiva, exercida pelo Departamento de Gestão e Incorporação de Tecnologias em Saúde (DGITS) é responsável pela gestão e coordenação das atividades da CONITEC, bem como pela emissão de relatórios técnicos sobre a tecnologia avaliada, levando em consideração as evidências científicas, a avaliação econômica e o impacto da incorporação da tecnologia no SUS.

O Plenário é responsável pela recomendação sobre incorporação, exclusão ou alteração das tecnologias no âmbito do SUS, sobre a constituição ou alteração de protocolos clínicos e diretrizes terapêuticas e pela atualização da Relação de Medicamentos Essenciais (RENAME) $)^{2}$. Os membros do plenário são um representante de cada uma das setes secretarias do MS, o Conselho Federal de Medicina, o CNS, o Conselho Nacional de Secretários de Saúde (CONASS), o Conselho Nacional de Secretarias Municipais de Saúde (CONASEMS), a ANS e a ANVISA. Todas as recomendações emitidas pelo Plenário são submetidas à consulta pública. As contribuições e sugestões da consulta pública são organizadas e inseridas ao relatório final da CONITEC, que, posteriormente, é encaminhado para o Secretário de Ciência, Tecnologia e Insumos Estratégicos para a tomada de decisão. O Secretário da SCTIE pode, ainda, solicitar a realização de audiência pública antes da sua decisão.

\footnotetext{
${ }^{2}$ A Relação Nacional de Medicamentos Essenciais (Rename) é uma lista de medicamentos que deve atender às necessidades de saúde prioritárias da população brasileira. Deve ser um instrumento mestre para as ações de assistência farmacêutica no SUS. As listas de medicamentos essenciais são uma das estratégias da política de medicamentos da Organização Mundial da Saúde (OMS) para promover o acesso e uso seguro e racional de medicamentos.
} 
Considerando a importância da articulação entre os atores que atuam nos âmbitos de saúde e C\&T, este trabalho teve como objetivo avaliar o alinhamento entre as ações de financiamento à ciência, tecnologia e inovação no setor farmacêutico e a incorporação de tecnologias no SUS, de modo a constatar se o alinhamento e a relação entre as agências são suficientes.

Afinal, é ideal que os projetos da área da saúde financiados com recursos públicos deem origem a tecnologias que possam ser inseridas no SUS, para serem disponibilizadas para a sociedade, em especial às camadas mais carentes, de forma a cumprir o objetivo de desenvolvimento social e melhoria da saúde pública das agências de fomento. No entanto, tal análise não é trivial.

Primeiramente, porque há um grande espaço de tempo entre a execução do projeto apoiado e a inserção no mercado de um produto baseado na tecnologia ao qual ele deu origem. Considerando o tempo de desenvolvimento citado pela literatura, esse espaço pode ser de até 15 anos. Sendo assim, considerando-se que o fomento a inovação acontece de forma mais pungente no Brasil a partir de 2004, o ciclo de desenvolvimento das tecnologias apoiadas mesmo nos anos iniciais provavelmente ainda não está completo. Além disso, como há um grande risco associado aos projetos de inovação, pode ser que parte deles tenha simplesmente falhado. Ademais, o Sistema Brasileiro de Inovação ainda está em amadurecimento. Sendo assim, não se pode esperar um alinhamento estreito entre as instituições, em especial de áreas distintas como C\&T\&I e saúde. No entanto, todos os atores justificam suas ações com base em um objetivo em comum, de elevar o patamar competitivo do país e trazer desenvolvimento para a sociedade, e na área de saúde, é usado também o argumento de se ampliar o acesso da população às tecnologias. Considerando todos os fatores citados, cabe analisar se há uma consonância entre os agentes, mesmo que preliminar.

\section{METODOLOGIA}

Foi realizada uma pesquisa exploratória, por meio de análise documental qualitativa, baseada em informações de acesso público disponibilizadas no site das agências. Buscouse identificar e comparar os processos de avaliação e os critérios adotados pela FINEP no Edital Subvenção Econômica de 2006, 2007, 2008, 2009, e 2010, para a seleção de projetos de desenvolvimento de novas tecnologias a serem financiados na área de saúde e os critérios utilizados pela CONITEC para incorporação de tecnologias no SUS.

Optou-se por analisar esta agência e estes editais de financiamento pois são os que apresentam de forma mais explicita os critérios adotados, bem como contemplam um amplo horizonte de tempo. Ademais, a subvenção econômica é uma modalidade de apoio bastante almejada e procurada pelas empresas, uma vez que constitui no aporte de recursos não-reembolsáveis, exigindo apenas uma contrapartida fixa de acordo com o porte do proponente.

Foram analisados as áreas apoiadas em cada Edital e os critérios para a seleção de projetos, bem como a relação de tecnologias avaliadas pela CONITEC e sua área terapêutica, a fim de identificar se há semelhanças entre as tecnologias apoiadas e as candidatas para a incorporação no SUS. Além disto, foram comparados os critérios de forma qualitativa, a fim de verificar a sinergia entre as avaliações das agências.

\section{RESULTADOS E DISCUSSÃO}


O fluxo de avaliação de tecnologias da CONITEC se dá conforme ilustrado na FIGURA

1.

\section{FIGURA 1 - Fluxo de avaliação de tecnologias da CONITEC}

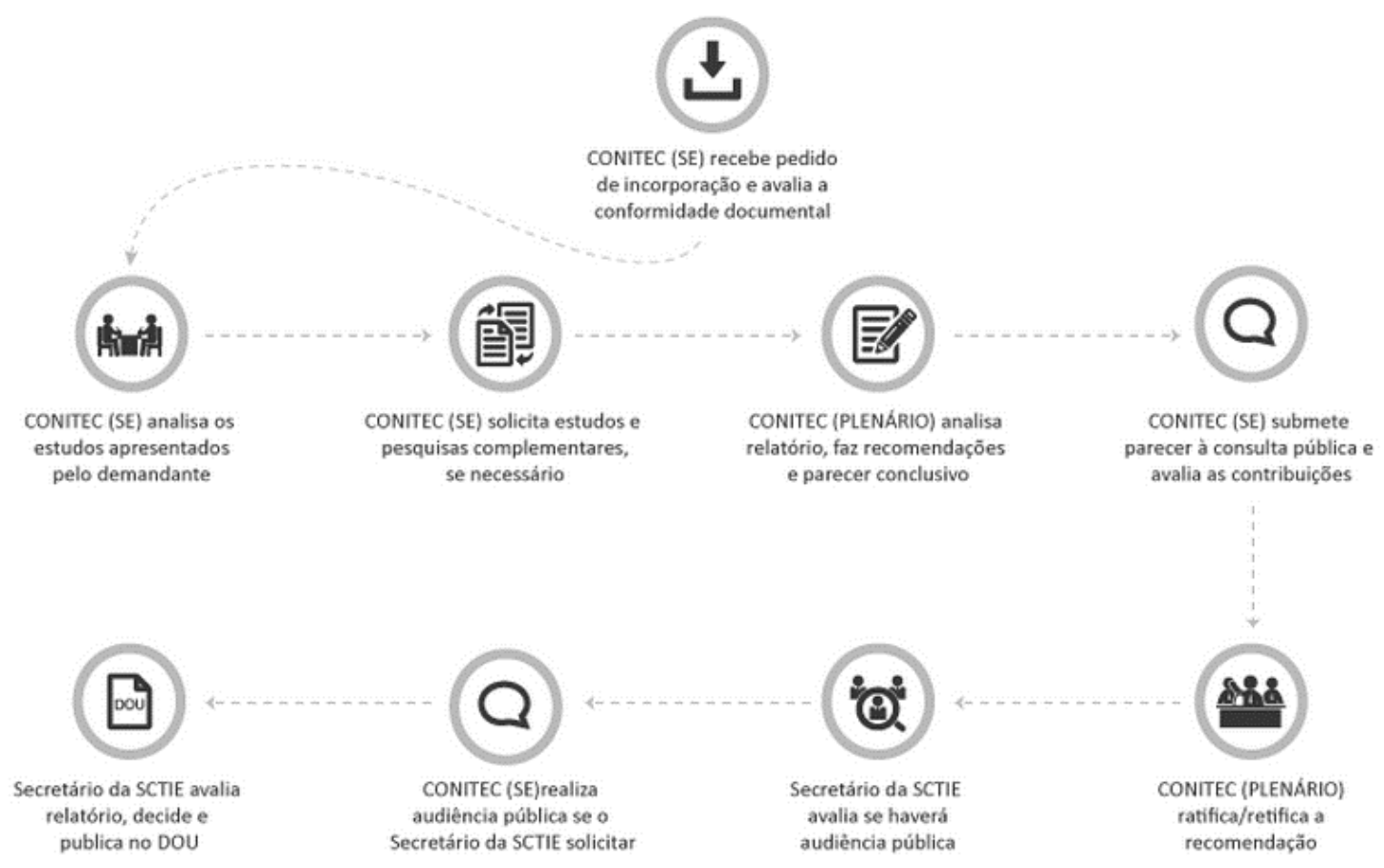

Fonte: CONITEC, 2016.

A proposta de incorporação de tecnologia em saúde deve conter as informações descritas no QUADRO 1. As informações exigidas incluem não só dados técnicos, como a descrição da condição de saúde tratada e da tecnologia, mas também avaliações econômicas na perspectiva do SUS e análise de impacto orçamentário. Além disso, as evidências científicas devem estar comparadas às tecnologias já disponibilizadas no SUS. Os critérios adotados pela CONITEC são critérios racionais e parâmetros de eficácia, efetividade, eficiência e segurança adequados às necessidades de saúde; e preconiza-se a incorporação de tecnologias que sejam relevantes para o sistema de saúde, baseadas na relação custo-efetividade. (BRASIL,2012)

Também é importante ressaltar que a incorporação de uma tecnologia é feita para condições ou doenças específicas. Sendo assim, um mesmo fármaco pode ser submetido a dois processos de avaliação diferentes, de acordo com a sua aplicação. O cetuximabe, por exemplo, foi objeto de dois relatórios da CONITEC, número 79 e 80 respectivamente, um em combinação com Folfiri ou Folfox no tratamento do câncer colorretal metastático KRAS selvagem com metástases hepáticas exclusivas irressecáveis, e outro para tratamento do carcinoma de células escamosas de cabeça e pescoço localmente avançado. 


\section{QUADRO 1 - Documentação necessária para solicitação de incorporação de tecnologia em saúde no SUS}

\begin{tabular}{|c|c|}
\hline Requisito & Descrição \\
\hline Ofício & $\begin{array}{l}\text { Deve estar na primeira página do processo e } \\
\text { conter a identificação do proponente, do } \\
\text { destinatário (CONITEC/SCTIE/MS), assunto } \\
\text { (nome da tecnologia e indicação solicitada), data } \\
\text { e local. }\end{array}$ \\
\hline Documentação do proponente & $\begin{array}{l}\text { Pessoa jurídica- Contrato Social da empresa } \\
\text { (cópia com firma reconhecida em cartório): } \\
\text { - Procuração do proponente (caso o responsável } \\
\text { pela assinatura da solicitação não conste no } \\
\text { Contrato Social da empresa); } \\
\text { Pessoa física: } \\
\text { - Cópias autenticadas do RG e CPF. }\end{array}$ \\
\hline Formulário & $\begin{array}{l}\text { O formulário deve ser submetido por meio do } \\
\text { Sistema para a Gestão Eletrônica de Processos de } \\
\text { Incorporação de Tecnologias no SUS (e-GITS). } \\
\text { Esse sistema permite a submissão de propostas de } \\
\text { incorporação, exclusão e alteração de tecnologias } \\
\text { em saúde. Existem duas categorias de } \\
\text { formulários, uma de órgãos/instituições não } \\
\text { vinculadas ao SUS e pessoas físicas e outro de } \\
\text { órgãos/instituiçõos do SUS. }\end{array}$ \\
\hline Documento principal & $\begin{array}{l}\text { Descrição da doença/condição de saúde } \\
\text { relacionada à utilização da tecnologia; } \\
\text { Descrição da tecnologia; } \\
\text { Descrição das evidências científicas da tecnologia } \\
\text { comparada à(s) disponibilizada(s) no SUS - } \\
\text { Revisão Sistemática ou Parecer Técnico- } \\
\text { Científico; } \\
\text { Estudo de avaliação econômica na perspectiva do } \\
\text { SUS; } \\
\text { Análise de impacto orçamentário; } \\
\text { Referências bibliográficas; } \\
\text { Anexo (cópia da bula ou instrução de uso } \\
\text { aprovada na ANVISA). }\end{array}$ \\
\hline Textos completos & $\begin{array}{l}\text { Dos estudos científicos referenciados em meio } \\
\text { digital. }\end{array}$ \\
\hline Documentos impressos contendo os artigos & $\begin{array}{l}\text { Em língua estrangeira, exceto inglês e espanhol, } \\
\text { com tradução juramentada para o português, se } \\
\text { for o caso. }\end{array}$ \\
\hline
\end{tabular}

Fonte: CONITEC, 2016

Historicamente, o processo de avaliação utilizado pela FINEP não é explicado de forma tão detalhada quanto o da CONITEC. Apenas em 2010, o Edital trouxe um maior detalhamento das etapas do processo de avaliação. Além disto, o processo de análise e os critérios de seleção do programa subvenção econômica variam de acordo com cada edital. De modo geral primeiramente é feita uma análise documental, para avaliar se todas as exigências formais foram cumpridas, e, em seguida, a proposta é analisada por comitês de especialistas, tanto da FINEP quanto de consultores externos. Os critérios adotados 
também variam, mas geralmente os requisitos da proposta abrangem critérios pertinentes ao mérito da inovação, os aspectos mercadológicos da inovação e à capacidade de execução e os aportes da empresa, e tem pesos distintos. As propostas avaliadas recebem notas de acordo com cada critério e são ranqueadas de modo a definir quais são aptas a seguir para as fases conclusivas e de homologação. O QUADRO 2 descreve os critérios definidos nos diferentes editais de subvenção.

\section{QUADRO 2 - Comparação dos critérios dos editais subvenção econômica nos diferentes anos}

\begin{tabular}{|c|c|}
\hline Critério & Edital \\
\hline Perspectivas de inserção no mercado dos resultados do projeto & 2006 \\
\hline Qualificação da equipe técnica do projeto & 2006 \\
\hline $\begin{array}{l}\text { Parcerias com instituições científicas e tecnológicas para o } \\
\text { desenvolvimento do projeto }\end{array}$ & 2006 \\
\hline Qualificação e proporção do aporte de contrapartida oferecido & 2006,2010 \\
\hline $\begin{array}{l}\text { Adequação das instalações de produção que serão utilizadas na } \\
\text { implementação da inovação }\end{array}$ & 2006 \\
\hline $\begin{array}{l}\text { Grau de integração e atribuições de cada uma das entidades partícipes } \\
\text { nos consórcios estabelecidos para desenvolvimento do projeto }\end{array}$ & 2006 \\
\hline Clareza e coerência da metodologia & 2006 \\
\hline $\begin{array}{l}\text { Consistência do orçamento, cronograma físico-financeiro e indicadores } \\
\text { de progresso do projeto }\end{array}$ & 2006, 2007 \\
\hline $\begin{array}{l}\text { Externalidades associadas ao projeto: adensamento de cadeias } \\
\text { produtivas, absorção de recursos humanos qualificados, } \\
\text { desenvolvimento local, social e ambiental }\end{array}$ & 2006 \\
\hline Grau de inovação do projeto & $2007,2008,2009,2010$ \\
\hline $\begin{array}{l}\text { Impactos esperados da inovação ou importância estratégica para a } \\
\text { sociedade }\end{array}$ & $2007,2008,2009,2010$ \\
\hline $\begin{array}{l}\text { Competência e experiência da empresa e capacidade técnica e } \\
\text { experiência da equipe executora }\end{array}$ & $2007,2008,2009,2010$ \\
\hline $\begin{array}{l}\text { Aderência ao tema, abrangência e efetividade do projeto na solução } \\
\text { dos problemas definidos no tema específico }\end{array}$ & $2008,2009,2010$ \\
\hline $\begin{array}{l}\text { Viabilidade técnica e financeira e adequação do orçamento do projeto } \\
\text { proposto }\end{array}$ & 2009 \\
\hline Plano de negócios & 2010 \\
\hline
\end{tabular}

Fonte: Elaboração própria, com base em FINEP, 2015.

É importante notar que os editais analisados são de um período anterior à criação da CONITEC em 2011. Mesmo assim, é pertinente analisar se os critérios utilizados pelas fontes de fomento à inovação estão alinhados aos princípios e necessidades do SUS, já que o fluxo de incorporação de tecnologias foi estabelecido em 2006 (BRASIL, 2006) e, 
desde então, já determinava que a deliberação fosse feita com base na relevância e no impacto da incorporação da tecnologia no SUS, baseado nos critérios citados acima e sempre em comparação com as tecnologias já incorporadas no sistema. Isso é importante, pois é desejável que as tecnologias financiadas pelas agências de fomento público estejam alinhadas às necessidades do sistema público de saúde, e sejam passíveis de incorporação por este no futuro.

Há pontos de convergência entre os critérios adotados pela CONITEC e pela FINEP. Ambos consideram os impactos no mercado da nova tecnologia e exigem a comparação de seus benefícios com outras semelhantes já disponíveis. No entanto, a análise do impacto do novo produto, a ser desenvolvido no projeto apoiado, no mercado e a importância estratégica para a sociedade deve ser realizada sob o ponto de vista do SUS, e não apenas do mercado privado. Já que os especialistas externos são envolvidos no processo de seleção dos projetos pela FINEP, é interessante que pelo menos um deles seja um profissional do SUS, ou possa trazer esta perspectiva para a análise. Além disso, ao se selecionar e priorizar os projetos, deve-se levar em conta o déficit da balança comercial de produtos do complexo econômico-industrial da saúde, de modo que as novas tecnologias desenvolvidas contribuam para a diminuição deste, que é um dos grandes gargalos do sistema de saúde brasileiro. A análise de mercado e a comparação com outras tecnologias exigida para seleção dos projetos para financiamento devem levar em conta o mercado público e as tecnologias já disponibilizadas no SUS.

Não se espera que os critérios adotados pela FINEP e as outras agências públicas de fomento à inovação sejam os mesmos adotados pela CONITEC. Isto seria inviável, pois o estágio de desenvolvimento das tecnologias avaliadas é muito diferente. Enquanto as tecnologias financiadas em projetos de inovação encontram-se em estágio preliminar de desenvolvimento, sendo candidatos a fármacos que por vezes não passaram ainda nem por testes pré-clínicos, a incorporação de tecnologias no SUS pressupõe que elas já estejam devidamente registradas na ANVISA, o que significa que já passaram por todas as etapas de desenvolvimento, incluindo testes clínicos de fase 3.

No entanto, é recomendável um alinhamento entre a FINEP e as outras agências de fomento e o SUS, no caso de tecnologias para saúde, pelo menos para que sigam as mesmas diretrizes e princípios. Isto se faz importante por diversos motivos. Primeiro, para que as agências públicas de fomento possam cumprir sua missão de promover o desenvolvimento social do Brasil, ao estimular o desenvolvimento de tecnologias em saúde que serão disponibilizadas para a maior parte da população, em especial para a parcela mais carente, através do SUS. Segundo, pois o Estado é um dos principais compradores de medicamentos de alto custo no Brasil, grupo que inclui em grande parte medicamentos inovadores. Sendo assim, para que a tecnologia financiada pelo Estado seja inserida com sucesso no mercado brasileiro, ela certamente deverá ser incorporada pelo SUS, e para tal deve obedecer aos critérios estabelecidos pela CONITEC.

O QUADRO 3 apresenta, através de exemplos, que tecnologias avaliadas pela CONITEC já foram passíveis de enquadramento em temas apoiados pelos editais de subvenção.

\section{QUADRO 3- Comparação das áreas temáticas dos editais subvenção econômica e tecnologias avaliadas pela CONITEC}

\begin{tabular}{|c|c|c|}
\hline Edital & $\begin{array}{c}\text { Áreas temáticas para apoio a projetos na } \\
\text { área de fármacos para saúde humana }\end{array}$ & $\begin{array}{c}\text { Exemplos de tecnologias análogas } \\
\text { avaliadas pela CONITEC }\end{array}$ \\
\hline
\end{tabular}




\begin{tabular}{|c|c|c|}
\hline Subvenção 2006 & $\begin{array}{l}\text { Fármacos e medicamentos: foco em AIDS e } \\
\text { Hepatite } \\
\text { - Obtenção de novas rotas de síntese que } \\
\text { possibilitem avanços tecnológicos na produção } \\
\text { do fármaco AZT; } \\
\text { - Desenvolvimento de moléculas inéditas } \\
\text { (análogos), farmacologicamente ativas e } \\
\text { comparáveis aos fármacos: Lopinavir, } \\
\text { Nelfinavir, Efavirenz e Tenofovir; } \\
\text { - Desenvolvimento de moléculas inéditas, } \\
\text { farmacologicamente ativas, e que se destinem } \\
\text { ao tratamento de Hepatite C. }\end{array}$ & $\begin{array}{l}\text { - Boceprevir e Telaprevir (Hepatite } \\
\text { C); } \\
\text { - Estavudina, indinavir, } \\
\text { maraviroque (antiretrovirais). }\end{array}$ \\
\hline Subvenção 2007 & $\begin{array}{l}\text { Inovações nas áreas de biodiversidade, } \\
\text { biotecnologia e saúde: } \\
\text { - Desenvolvimento, por rota biotecnológica, de } \\
\text { novos produtos antibióticos, antifúngicos e } \\
\text { antitumorais, e de substâncias bioativas da } \\
\text { biodiversidade brasileira; } \\
\text { - Desenvolvimento de novas biomoléculas e } \\
\text { fármacos, por rota biotecnológica, contra } \\
\text { hanseníase, leishmaniose tegumentar } \\
\text { americana, leishmaniose visceral (calazar), } \\
\text { esquistossomose, malária, tuberculose, câncer, } \\
\text { chagas e dengue. }\end{array}$ & $\begin{array}{l}\text { - Mesilato de imatinibe } \\
\text { (antitumoral). }\end{array}$ \\
\hline Subvenção 2008 & $\begin{array}{l}\text { Saúde: } \\
\text { - Desenvolvimento de moléculas com alto } \\
\text { potencial no campo sanitário e/ou impacto } \\
\text { nos gastos do SUS: somatotrofina, insulina } \\
\text { humana, calcitonina, hormônio liberador do } \\
\text { hormônio luteinizante (LHRH), } \\
\text { somatostatina, gonadotrofina coriônica } \\
\text { (HCG) e sérica (PMSG), hormônio folículo } \\
\text { estimulante humano (FSH), fator de } \\
\text { crescimento insulina dependente (IGF-I), } \\
\text { glucagon, filgrastima, glucocerebrosidase, } \\
\text { anticorpos monoclonais. }\end{array}$ & $\begin{array}{l}\text { - Rituximabe, cetuximabe } \\
\text { (anticorpos monoclonais). }\end{array}$ \\
\hline Subvenção 2009 & $\begin{array}{l}\text { Saúde: } \\
\text { - Desenvolvimento de fármacos e/ou } \\
\text { biofármacos, com foco nos seguintes } \\
\text { produtos: anticorpos monoclonais; } \\
\text { budesonida; ciclosporinas; estatinas; fator de } \\
\text { crescimento insulina dependente (IGF-I); } \\
\text { filgrastima; glucagon; glucocerebrosidase; } \\
\text { gonadotrofina coriônica (HCG) e sérica } \\
\text { (PMSG); hormônio folículo estimulante } \\
\text { humano (FSH); novas biomoléculas e } \\
\text { fármacos, produzidos por rota biotecnológica, } \\
\text { para doenças virais, doenças negligenciadas e } \\
\text { neoplasias. }\end{array}$ & $\begin{array}{l}\text { - Erlotinibe (antineoplásico); } \\
\text { - Medicamentos para tratamento da } \\
\text { doença pulmonar obstrutiva } \\
\text { crônica (DPOC). }\end{array}$ \\
\hline Subvenção 2010 & $\begin{array}{l}\text { Saúde: } \\
\text { - Desenvolvimento de inovações em moléculas } \\
\text { e processos que contribuam para o } \\
\text { desenvolvimento da produção nacional de }\end{array}$ & $\begin{array}{l}\text { - Natalizumabe (esclerose } \\
\text { múltipla); } \\
\text { - Fingolimode (esclerose múltipla). }\end{array}$ \\
\hline
\end{tabular}




\begin{tabular}{|l|l|l|}
\hline & $\begin{array}{l}\text { insumos farmacêuticos ativos e } \\
\text { medicamentos para uso no tratamento de } \\
\text { doenças infecciosas, degenerativas e } \\
\text { genéticas. }\end{array}$ & \\
\hline
\end{tabular}

Fonte: Elaboração própria, com base em FINEP e CONITEC,2015.

\section{CONCLUSÃO}

É necessária uma articulação virtuosa entre as demandas industriais e as necessidades de saúde de uma população, de modo a impulsionar o desenvolvimento econômico e social de um país. (VIANA; ELIAS, 2007) A articulação entre as políticas de desenvolvimento produtivo e a inovação e políticas de incorporação de tecnologias no sistema público de saúde seria vantajosa tanto para o desenvolvimento do setor produtivo de fármacos e medicamentos quanto do sistema de atenção à saúde. (KLEIN; HASENCLEVER; MACHADO, 2011). No caso brasileiro, para a plena execução do SUS é necessário a realização não só de políticas sociais e de saúde pública, mas também econômicas e de inovação, fato reconhecido já na lei orgânica da saúde, que coloca que o dever do Estado de garantir a saúde consiste na formulação e execução de políticas econômicas e sociais que visem à redução de riscos de doenças e de outros agravos. Além disto, o Estado deve estabelecer condições que assegurem acesso universal e igualitário às ações e aos serviços. (BRASIL, 1990)

Todavia, para que isto aconteça é necessário um alinhamento entre estas políticas sociais e econômicas e uma articulação entre atores responsáveis por sua implementação. (GUIMARÃES, 2006) Isto representa um sistema de inovação maduro, capaz não só de competir a nível internacional, mas também de favorecer o desenvolvimento e bem estar aos níveis regional e nacional.

Como o Sistema Nacional de Inovação é composto por diversos atores e suas interações, assim como o Sistema Setorial da Saúde, é importante que o Estado assuma a governança das ações e das políticas. O Ministério da Saúde (MS) pode assumir este papel, não só por realizar ações de organização dos serviços de saúde, mas também de financiador do desenvolvimento de novas tecnologias, seja diretamente seja através de convênio com outros órgãos como a FINEP, mas também como principal consumidor das tecnologias desenvolvidas. O MS poderia aproximar as agendas da pesquisa em saúde da política de saúde pública, aportar recursos para pesquisa em saúde, seja através de aporte direto ou por meio do seu poder de compras e pode ainda estabelecer prioridades de pesquisa fundamentadas nas necessidades da saúde da população. (GUIMARÃES, 2006) Para além disto, um sistema universal de saúde funciona como ferramenta organizadora das demandas de inovação da sociedade, que devem corresponder a necessidades não atendidas e também priorizadas, e das respostas de pesquisa em saúde a serem desenvolvidas (GUIMARÃES, 2013), já que fazem parte do sistema tanto as instituições reguladoras, quanto as de pesquisa e de produção de insumos, bem como os prestadores de serviços.

Os agentes das políticas de saúde e econômica devem trabalhar em sintonia pois não se pode tratar estas duas dimensões de forma independente ${ }^{3}$, sob o risco de insucesso de ambos os lados. O tratamento em separado, voltado para a proteção dos interesses e da pressão da indústria, que se utiliza da sociedade civil, para a absorção de novos produtos e processos no sistema, inadvertidamente faz com que as forças sociais, que 
historicamente vêm lutando por um sistema de saúde amplo e inclusivo no Brasil, acabem atuando de forma a prejudicar este objetivo, ao sobrecarregarem o sistema desconsiderando sua capacidade de financiamento e o embasamento científico das decisões de incorporação.

É indispensável então esta visão conjunta dos aspectos relacionados a promoção da inovação e a garantia da saúde pública tanto na análise das políticas envolvidas quanto no planejamento e articulação das ações dos atores.

\section{REFERÊNCIAS}

ALBUQUERQUE, E. DA M. Produção científica e sistema nacional de inovação. Ensaios FEE, v. 19, n. 1, p. 156-180, 1998.

BRASIL, Financiadora de estudos e projetos -FINEP [Internet]. A FINEP. [acesso em 2016 Set 5]. Disponível em http://www.finep.gov.br/.

BRASIL, Financiadora de Estudos e Projetos - FINEP [Internet]. Subvenção econômica. [acesso em 2016 Set 5]. Disponível em http://www.finep.gov.br/apoio-efinanciamento-externa/instrumentos-de-apoio/subvencao-economica.

BRASIL. Financiadora de Estudos e Projetos - FINEP [Internet]. Chamadas públicas do programa Subvenção Econômica dos anos 2006, 2007, 2008, 2009 e 2010 [acesso em 2016 Set 5]. Disponíveis em http://www.finep.gov.br/chamadas-publicas/.

BRASIL, Ministério da Saúde. Comissão Nacional de Incorporação de tecnologias no SUS - CONITEC [Internet]. [acesso em 2016 Set 5] Disponível em http://conitec.gov.br/.

BRASIL. Ministério da Saúde. Portaria No 2.009/GM de 13 de setembro de 2012

BRASIL. Ministério da Saúde. Portaria No 152/GM de 19 de janeiro de 2006 e Portaria No 3.323 de 27 de dezembro de 2006. Diário Oficial da União 2006.

BRASIL. Lei no 8.080 de 19 de Setembro de 1990. Dispõe sobre as condições para a promoção, proteção e recuperação da saúde, a organização e o funcionamento dos serviços correspondentes e dá outras providências. Diário Oficial da União 1990; 19 set.

CAPANEMA, L. X. DE L.; PALMEIRA FILHO, P. L. A cadeia farmacêutica e a política industrial: uma proposta de inserção do BNDES. 2004.

GADELHA, C. A. G. Desenvolvimento, complexo industrial da saúde e política industrial. v. 40, p. 11-23, 2006.

GUIMARÃES, R. Pesquisa em saúde no Brasil: Contexto e desafios. Revista de Saúde Pública, v. 40, p. 3-10, 2006.

GUIMARÃES, R. Pesquisa Translacional: uma interpretação. Ciência \& Saúde Coletiva, v. 18, n. 6, p. $1731-1744,2013$.

KLEIN, H. E.; HASENCLEVER, L.; MACHADO, C. J. S. Regulação e difusão de tecnologias e sua influência na capacitação tecnológica em saúde dos países em desenvolvimento. Revista Brasileira de Ciência, Tecnologia e Sociedade, v. 2, n. 2, p. p.130-149, dez. 2011. 
LEONEL, S. G. et al. Understanding financial innovationsystems: Veblen and Minskyat the periphery. European Journal of Economics and Economic Policies: Intervention, v. 10, n. 1, p. $93-105,2013$.

MALERBA, F. Sectoral Systems: How and Why Innovation Differs across Sectors. [S.1: s.n.], 2006.

Disponível

em:

<http://www.oxfordhandbooks.com/view/10.1093/oxfordhb/9780199286805.001.0001/ oxfordhb-9780199286805-e-14>.

NOVAES, H. M. D.; ELIAS, F. T. S. Uso da avaliação de tecnologias em saúde em processos de análise para incorporação de tecnologias no Sistema Único de Saúde no Ministério da Saúde. Cadernos de Saúde Pública, v. 29, n. Sup, p. s7-s16, 2013.

TEIXEIRA, A. L. S. ; RAPINI, M.S. ; SZAPIRO, M. . Discurso e Prática da Política Industrial no período 2005-2014: uma avaliação da distribuição setorial dos recursos reembolsáveis do BNDES e da FINEP. In: Anais do II Encontro Nacional da Economia Industrial e Inovação. Rio de Janeiro: ABEIN, 2017.

VIANA, A. L. D.; ELIAS, P. E. M. Saúde e desenvolvimento. Healthcare and development. Ciência \& Saúde Coletiva, v. 12, p. 1765-1777, 2007. 